\title{
Left Ventricular Twist and Shear in Patients With Primary Mitral Regurgitation
}

\author{
Meral Reyhan, $\mathrm{PhD}^{1,2}$, Zhe Wang, MS $^{2,3}$, Ming Li, BS ${ }^{4}$, Hyun J. Kim, $\mathrm{PhD}^{3}$, Himanshu \\ Gupta, MD ${ }^{5,6}$, Steven G. Lloyd, MD ${ }^{5,6}$, Louis J. Dell'Italia, MD ${ }^{5,6}$, Thomas Denney, PhD $^{4}$, and \\ Daniel B. Ennis, PhD ${ }^{1,2,3, *}$
}

${ }^{1}$ Biomedical Physics Interdepartmental Program, University of California, Los Angeles, California, USA; ${ }^{2}$ Department of Bioengineering, University of California, Los Angeles, California, USA; ${ }^{3}$ Department of Radiological Sciences, University of California, Los Angeles, California, USA; ${ }^{4}$ Department of Electrical and Computer Engineering, Auburn University, Auburn, Alabama, USA; ${ }^{5}$ Department of Medicine, Division of Cardiovascular Disease, University of Alabama at Birmingham, Birmingham, Alabama, USA; and ${ }^{6}$ Birmingham VA Medical Center, Birmingham, Alabama, USA

\begin{abstract}
Purpose: To evaluate the relationship between left ventricular (LV) twist, shear, and twist-pervolume and the severity of mitral regurgitation (MR). Primary MR is a valvular disorder that induces LV dysfunction. There exist several measures of LV rotational mechanics, but it remains unclear which measure of LV dysfunction best accords with the severity of MR. We hypothesized that LV systolic twist-per-volume slope would decrease with increasing severity of MR because of both decreases in rotational mechanics and increases in stroke volumes.
\end{abstract}

Materials and Methods: Normal subjects $(n=54)$, moderate MR patients $(n=29)$, and severe MR patients ( $n=54)$ were studied. Magnetic resonance imaging (MRI) was performed on a $1.5 \mathrm{~T}$ scanner and grid-tagged LV images were collected at the LV base and LV apex. Measures of LV rotational mechanics were derived from tagged images using Fourier Analysis of STimulated echoes (FAST).

Results: Peak systolic twist-per-volume slope was significantly different for all pairwise comparisons $(P<0.0001)$ and compared to normal subjects $(-0.14 \pm 0.05 \% \mathrm{~mL})$ was decreased in moderate MR $(-0.12 \pm 0.04 \% \mathrm{~mL})$ and further decreased in severe MR $(-0.07 \pm 0.03 \% \mathrm{~mL})$.

Conclusion: Peak systolic twist-per-volume slope significantly decreased with increasing severity of MR and is therefore a suitable quantitative imaging biomarker for LV dysfunction in patients with MR.

Primary mitral regurgitation (MR) is a common valvular disorder that induces left ventricular (LV) dysfunction. ${ }^{1}$ Primary MR is characterized by an incomplete closure of the mitral valve, which permits the flow of blood across the mitral valve during systole

\footnotetext{
*Address reprint requests to: D.B.E., 10945 LeConte Ave., Ste. 1417, Los Angeles, CA 90095-7206, Daniel.Ennis@ucla.edu. View this article online at wileyonlinelibrary.com. DOI: $10.1002 /$ jmri.24811
} 
(decreased afterload) and leads to increased end-diastolic volume (increased preload). Additionally, chronic MR is known to deleteriously impact the rotational dynamics of the $\mathrm{LV}^{2-4}$ There exist several measures of the rotational mechanics of LV performance, but it remains unclear which measures are best for characterizing deleterious changes in LV function. ${ }^{5-7}$ The twisting motion of the heart arises from transmural differences in the local myofiber orientation, which is thought to minimize transmural stress gradients. ${ }^{8}$ Hence, alterations in the twisting mechanics may be indicative of incipient worsening disease. Several measures of rotational mechanics are suggested as imaging biomarkers for LV dysfunction, ${ }^{9,10}$ including twist, twist-per-volume, and shear angle.

LV twist is the preferred nomenclature for the measurement defined as the difference in the rotation of the apex relative to that of the base of the heart. ${ }^{9}$ A decrease in LV twist during the progression of MR has previously been evaluated with ultrasound ${ }^{2-5}$ in human subjects and implanted radiopaque markers in animal studies. ${ }^{6,7}$ Twist-per-volume slope normalizes for the volume of ejected blood. Therefore, a change represents a disruption of the fundamental connection between myofiber shortening and ejection. CL-shear is a measure of rotational mechanics that accounts for changes in LV ventricular dimensions, which is reported to increase with MR. ${ }^{11}$

LV rotational mechanics is a potentially valuable biomarker of LV dysfunction evaluation. Traditional analysis methods like FindTags ${ }^{12}$ require significant user interaction time ( $\sim 1$ hour) and the best alternatives typically require several tens of minutes, which significantly limits their clinical adoption. Fourier Analysis of STimulated Echoes (FAST) is a new Magnetic resonance imaging (MRI) tissue tagging method that has recently been shown to compare favorably with conventional estimates of $\mathrm{LV}^{13}$ twist from cardiac tagged images, but with significantly reduced user interaction time (2-3 minutes primarily for study selection). ${ }^{14}$ FAST was applied to grid tag images acquired from patients with moderate and severe degenerative isolated MR to evaluate LV twist, CL-shear angle, and systolic twistpervolume slope. We hypothesized that LV systolic twist-pervolume slope would decrease with increasing severity of MR because it accounts for decreases in both rotational mechanics and increases in stroke volumes.

\section{Materials and Methods}

The local Institutional Review Board approved this study and all subjects provided written informed consent. Moderate MR patients $(n=29)$ and severe MR patients $(n=54)$ with degenerative isolated MR were studied with MRI. Clinicians judge categories of moderate or severe MR by color flow Doppler, LV ejection fraction (EF) of more than 55\%, LV endsystolic dimension of $40 \mathrm{~mm}$, echocardiographic thickening of the mitral valve leaflets, and prolapse.

Healthy normal subjects $(n=54)$ with no history of cardiovascular disease were studied as age-matched controls for the MR patients. MR severity was diagnosed qualitatively with echocardiography and Doppler studies and quantitatively with cine MRI. Subject demographics are summarized in Table 1. Data from patients in the normal subject and severe MR subject groups have been presented in a previous study. ${ }^{11}$ 
Breath-held electrocardiogram (ECG)-triggered images were acquired for all subjects on a 1.5T scanner (Signa, GE Healthcare, Milwaukee, WI). Prospectively triggered balanced steady-state free precession (bSSFP) cine images were collected in parallel $\mathrm{LV}^{13}$ shortaxis planes using the following typical parameters: $40 \times 40 \mathrm{~cm}$ field of view, $256 \times 128$ matrix size, $8 \mathrm{~mm}$ slice thickness, $45^{\circ}$ flip angle, $1.6 / 3.8 \mathrm{msec}$ echo/repetition times, $977 \mathrm{~Hz} /$ pixel receiver bandwidth, and 20 cardiac phases per slice in a single breath-hold.

A prospectively triggered spoiled gradient echo sequence was used to acquire short-axis tagged images of the LV with the following typical parameters: $40 \times 40 \mathrm{~cm}$ field-of-view, $256 \times 128$ acquisition matrix, $8 \mathrm{~mm}$ slice thickness, $10^{\circ}$ imaging flip angle, $4.2 / 8.0 \mathrm{msec}$ echo/repetition times, $244 \mathrm{~Hz} /$ pixel receiver bandwidth, 20.4 to $51.1 \mathrm{msec}$ temporal resolution, and 20 cardiac phases per slice in a single breath-hold. Grid tags were oriented $45^{\circ}$ relative to the frequency encoding axis with $7 \mathrm{~mm}$ tag spacing.

An experienced trainee used the following criteria to select short axis slices and derive LV twist measurements: 1) the most apical slice containing the presence of the blood pool throughout the entire cardiac cycle, and 2) the most basal slice in which the LV myocardium maintained a continuous annular shape during the entire cardiac cycle were selected.

\section{Fourier Analysis of STimulated echoes (FAST)}

FAST $^{14}$ is a semiautomated and validated image processing method that only requires 2-3 minutes of user interaction time (mostly for study selection) to quantify LV rotational mechanics. FAST determines object rotation in Fourier space, where rotation in tagged MR images is easier to track. User-defined contours of the epicardium in an end-systolic cardiac frame and endocardium in an early systolic cardiac frame were used to isolate the LV myocardium. The central frequency information in Fourier space was nulled and the Fourier image was symmetrically cropped in $k$-space beyond the first harmonic Fourier peak to reduce computational times, then cumulative rotation of the ventricle was determined with 2D cross-correlation. ${ }^{15}$ This series of image processing steps was applied to both the apical and basal images of the heart, and the difference between the two rotations was defined as twist. The FAST method was previously validated using line tag images in healthy volunteers. ${ }^{14}$ Peak twist was defined as the maximum difference in degrees of rotation $(\phi)$ between the apex and the base:

$$
\text { Peak Twist }=\operatorname{Max}\left(\phi_{\text {apex }}-\phi_{\text {base }}\right)
$$

The systolic twist-per-volume slope was defined as peak LV twist divided by the difference between LV end-systolic volume and end-diastolic volume.

$$
\text { Twist-per-volume }=\frac{\text { Peak Twist }}{\text { LVESV-LVEDV }}
$$

The circumferential-longitudinal shear-angle (CL-shear angle) was defined as 9,16 : 


$$
C L-\text { Shear }=\frac{\phi_{\text {apex }} \rho_{\text {apex }}-\phi_{\text {base }} \rho_{\text {base }}}{D}
$$

where $\rho$ is the epicardial radius of the apex or base in millimeters, and D is the distance between the apical and basal in millimeters. CL-shear angle is proposed as a measure of LV rotational mechanics that accounts for differences in heart size and the location of the apical and basal slices used for analysis. ${ }^{17}$ Estimates of apical and basal epicardial radii were made from averaging the semimajor with the semiminor axes of ellipses fit to the user-defined epicardial contours. The twist rate was defined as the discrete derivative of twist with respect to time with units of degrees-per-second (deg/s). Peak twist rate was defined as the maximum twist rate. Peak untwist rate was defined as the minimum twist rate. Peak normalized untwist rate was defined as the minimum twist rate divided by peak twist as per Thompson et al. ${ }^{13}$ During ejection the volume of the LV decreases, while twist increases, which leads to a negative systolic twist-per-volume slope. ${ }^{7}$

Estimates of LV volumes were obtained from epicardial and endocardial contours manually traced at end-systole and end-diastole from the bSSFP images by a trainee with more than 3 years experience. Papillary muscles were included in the LV volume. The contours were then propagated to the rest of the temporal frames with a dual propagation technique. ${ }^{18} \mathrm{LV}$ volumes were computed by summing the areas defined by the endocardial contours in each short-axis slice multiplied by slice thickness. ${ }^{18} \mathrm{LV}$ mass was computed by summing the areas between endocardial and epicardial contours multiplied by slice thickness and myocardial density $(1.05 \mathrm{~g} / \mathrm{mL}) .{ }^{13}$

\section{Statistical Analysis}

Summary statistics of the rotational mechanics measurements were reported for each group. Analysis of variance (ANOVA) ${ }^{19}$ was used to test for differences in peak twist, apical rotation, basal rotation, systolic twist rate, diastolic untwisting rate, systolic twist-pervolume slope, distance between apical and basal slices, and apical and basal epicardial radii between normal, moderate MR, and severe MR groups while adjusting for the covariates of age, weight, height, and gender. A similar analysis was also performed for peak CL-shear angle (due to some subject height and gender not being recorded an additional ANOVA test was performed without subject and gender as part of the sensitivity check). Additionally, a one-way ANOVA with three groups (normal, moderate MR, and severe MR) was used to assess differences in time to peak twist, peak normalized untwisting rate, endocardial radii, and the demographic values in Table 1. Multiple comparisons (Tukey's least significant difference [LSD] procedure) were applied to each measurement to determine which groups were significantly different from each other. $P<0.05$ was considered statistically significant. All statistical analyses were performed with MatLab v. 7.10 (MathWorks, Natick, MA). 


\section{Results}

\section{Patient Demographics}

Patient demographics are summarized in Table 1. Significant differences between the age, gender, height, end-systolic volume, end-diastolic volume, and LVED mass of the normal, moderate MR, and severe MR subjects were detected with a three group one-way ANOVA. No significant differences were noted in weight and heart rate with $95 \%$ confident intervals. Significant increases in end-systolic volume, end-diastolic volume, and LVED mass were expected and observed for MR patients.

\section{Image Quality}

Figure 1 represents the typical tagged image quality from normal, moderate MR, and severe MR subjects at the time of peak twist for the apex and base. The peak twist, apical rotation, and basal rotation corresponding to the images are also reported in the figure. Due to $T_{1}$ relaxation tag contrast fades at middle and late diastolic phases, making the measurement of LV diastolic rotational mechanics challenging.

\section{Temporal Resolution}

The temporal resolution for normal subjects, patients with moderate MR, and patients with severe MR were $33.2 \pm 7.2 \mathrm{msec}, 30.9 \pm 5.2 \mathrm{msec}$, and $31.6 \pm 5.9 \mathrm{msec}$, respectively, and are not significantly different per ANOVA.

\section{Rotation}

LV apical and basal peak rotation measures are summarized in Table 2. Mean peak apical rotation for normal subjects, moderate MR patients, and severe MR patients were: 7.5 $\pm 3.6^{\circ}, 5.8 \pm 2.7^{\circ}$, and $6.2 \pm 2.8^{\circ}$ respectively. The ANOVA of peak apical rotation between the groups showed differences among the groups $(P=0.004)$ and with respect to the covariate of age $(P=0.005)$. LSD for apical rotation showed a significant difference between the normal subject group and the moderate MR patients and between the normal subject group and the severe MR patients.

The ANOVA of peak basal rotation between the groups showed differences among the groups $(P=0.04)$ and with respect to age $(P=0.004)$. LSD for basal rotation detected differences between the normal subject group and the severe MR patients and between the moderate MR patients and the severe MR patients.

\section{Twist Analysis}

LV apical and basal rotation, LV twist and twist rates, and LV twist-per-volume results are summarized in Table 2. Figure 2 demonstrates the changes in mean LV twist for normal, moderate MR, and severe MR subjects. Figure 3 is a scatterplot that demonstrates the peak LV twist data from normal, moderate MR, and severe MR subjects.

Similar ANOVA results were observed for the mean peak LV twist, mean peak LV systolic twist rate, and mean diastolic untwisting rate, where significant differences were observed between normal subjects and MR patients. However, no significant difference was observed 
between moderate and severe MR patients for these measures. No significant differences were observed due to patient demographic covariates (age, weight, height, gender). Normalized peak untwisting rate and time to peak twist showed no significant difference among the three groups.

\section{Systolic Twist-per-Volume}

Systolic twist-per-volume slope for normal subjects, moderate MR patients, and severe MR patients was: $-0.14 \pm 0.05 \% \mathrm{~mL},-0.12 \pm 0.04 \% \mathrm{~mL}$, and $-0.07 \pm 0.03 \% \mathrm{~mL}$, respectively. The ANOVA of systolic twist-per-volume slope between the groups showed differences among the groups $(P<0.0001)$ and with respect to weight $(P=0.006)$. LSD detected significant decreases between normal subjects and moderate MR patients, normal subjects and severe MR patients, and moderate MR patients and severe MR patients. These differences were not associated with patient demographic covariates (age, weight, height, gender). Figure 4 illustrates the twist-per-volume data for normal, moderate MR, and severe MR subjects.

\section{CL-Shear Angle}

CL-shear angle results and the measured parameters used to estimate CL-shear are summarized in Table 3. Mean peak CL-shear angle for normal subjects, moderate MR patients, and severe MR patients were: $5.0 \pm 1.4^{\circ}, 4.7 \pm 1.6^{\circ}, 5.0 \pm 1.3^{\circ}$, respectively. Additionally, the ANOVA of peak CL-shear angle did not reveal any differences between the three groups $(P=0.7)$. Figure 5 shows the $\mathrm{CL}$-shear angle results for normal, moderate MR, and severe MR subjects. Figure 6 illustrates the peak CL-shear data for normal, moderate MR, and severe MR subjects.

In order to investigate the underlying reason that CL-shear angle measures were similar across the groups, the individual measures used to evaluate CL-shear angle were investigated (Table 3). LSD showed significant increases between the normal subject group and the severe MR patients and between the moderate MR patients and the severe MR patients for the apical and basal radii. LSD did not detect significant differences between any pairwise comparison of normal subjects, moderate MR patients, and severe MR patients for the distance between apical and basal slices.

A Hedges' effect size test comparing healthy subjects and patients with moderate MR shows peak twist-per-volume slope $(g=0.50)$, LVESV $(g=0.27)$, LVEDV $(g=0.34)$, LVED mass $(\mathrm{g}=0.20)$. Similarly, when comparing patients with moderate MR and patients with severe MR the effect size values are: peak twist-per-volume ( $g=1.67)$, LVESV $(g=0.98)$, LVEDV $(\mathrm{g}=1.22)$, LVED $(\mathrm{g}=1.25)$. The comparison shows that peak twist-per-volume slop has the largest effect size and thus higher discrimination power to detect differences between groups.

\section{Discussion}

LV twist was shown to decrease significantly in patients with moderate or severe MR. In moderate MR patients this decrease was primarily due to a significant decrease in apical twist, while in patients with severe MR there was a significant reduction in both basal and apical rotations. Ennis et $\mathrm{al}^{7}$ studied chronic "pure" MR in sheep using implanted 
radiopaque markers and demonstrated a significant decrease in both LV twist and in twistper-volume slope. Additionally, Tibayan et $\mathrm{al}^{6}$ studied the evolution from acute to chronic MR in dogs using videofluroscopy and radiopaque markers and also observed a decrease in twist after 3 months of chronic MR. Borg et $\mathrm{al}^{5}$ used speckle tracking echocardiography in patients with chronic primary MR to illustrate that the onset of LV untwist was delayed and untwisting rate was reduced in patients with MR. They did not observe significant differences in LV twist when compared to normal subjects and instead proposed the use of a twist-per-volume slope between peak twist and mitral valve opening, which showed a significant decrease in patients with MR. The expected decrease in peak twist paired with the increase in LV volume seen in patients with MR could make the twist-per-volume slope particularly insightful for evaluating the severity of dysfunction. Reduction in LV twist in patients with MR is consistent with previous reports. ${ }^{4,7,20}$ The decrease in apical rotation may be due to spherical remodeling of the mid-ventricular and apical LV. ${ }^{11}$ There were not, however, significant differences in peak LV twist between moderate and severe MR subjects, therefore peak LV twist does not correlate with the severity or progression of MR in this patient cohort. Both moderate and severe MR were associated with significant decreases in the peak untwisting rate, which are consistent with the previous literature. ${ }^{5}$ However, tag fading occurs around $500 \mathrm{msec}$ into the cardiac cycle, which makes measuring peak untwisting rate with the temporal resolution of this study challenging.

Peak LV CL-shear angle appeared to pseudo-normalize in patients with MR. The formula for CL-shear angle depends on several factors. The pseudo-normalization of CL-shear angle in moderate and severe MR can be explained by the significant increase in the apical and basal epicardial radii in conjunction with the significant decrease in apical rotation in MR patients compared with normal subjects. The increase in apical and basal epicardial and endocardial radii in the MR patient groups were consistent with the spherical global remodeling previously demonstrated by Schiros et al. ${ }^{11}$ The severe MR patients also had a significant decrease in basal rotation, which further pseudo-normalizes CL-shear angle compared to moderate MR and normal subjects. Additionally, the pseudo-normalization did not occur just for peak CL-shear angle, but was apparent throughout the cardiac cycle (Fig. $5)$.

In another study, CL-shear pseudo-normalizes with increasing age as a consequence of decreasing twist and increasing ventricular volumes. ${ }^{21} \mathrm{CL}$-shear angle may not be a suitable imaging biomarker for the detection of LV dysfunction.

For patients with MR, the pseudo-normalization obscured the presence of LV dysfunction and led to no significant difference between the normal subjects and the MR patients. CLshear angle may not be a suitable imaging biomarker for the detection of LV dysfunction in patients with MR, since the measure pseudo-normalizes as a consequence of pathologically increased LV volumes.

We also calculated the torsion-to-shortening ratio (TSR) ${ }^{22}$ for a subset of 20 normal subjects, 20 patients with moderate MR, and 20 patients with severe MR. TSR was unchanged across groups due to a decrease in torsion and a decrease in shortening. 
A significant decrease in the systolic twist-per-volume slope was detected for all pairwise comparisons of the subject groups (severe MR being the smallest), which is consistent with the literature. ${ }^{7}$ Importantly, twist-per-volume slope was the only measure of rotational mechanics that distinguished all three groups. The changes indicate that LV stroke volume increases with respect to MR severity, while twist decreases. This may indicate an energetically unfavorable ejection pattern and lead to exacerbation of the underlying disease. 8

\section{Limitations}

Patients and healthy subjects showed some differences in demographics, hence we cannot exclude that this influenced the observed changes in $\mathrm{LV}^{23}$ rotational mechanics. All studies were performed with a $1.5 \mathrm{~T}$ MR scanner, therefore diastolic rotational information was limited due to tag fading. Performing imaging with a 3T MR scanner or using a complementary tagging pattern could improve diastolic estimates. In future studies, the temporal resolution could be improved in order to refine estimates of diastolic rotational mechanics, but this can be difficult to achieve in patients with limited breath-hold capacity.

The FAST method has previously been validated using line tags, whereas grid tags were used for the reported study. Careful validation of FAST for grid tags is still needed, but as all subjects were imaged with grid tags and analyzed with the same FAST processing method, the comparison between groups is expected to be fair.

In conclusion, the results of this study have shown that systolic twist-per-volume slope was significantly decreased for moderate MR subjects and further decreased for severe MR subjects. Peak LV twist decreases similarly in patients with either moderate or severe MR compared with normal subjects. Therefore, peak systolic twist-per-volume slope may serve as quantitative imaging biomarkers for LV dysfunction in patients with MR.

\section{Acknowledgments}

Contract grant sponsor: American Heart Association and the National Institutes of Health National Heart, Lung, and Blood Institute; Contract grant numbers: AHA 11PRE6080005 (to M.R.), NIH/NHLIB R00-HL087614 (to D.E.), NIH/NHLIB grant P50-HL077100 (to T.D.).

\section{References}

1. Perloff JK, Roberts WC. The mitral apparatus. Functional anatomy of mitral regurgitation. Circulation 1972;46:227-239. [PubMed: 5046018]

2. Moustafa SE, Kansal M, Alharthi M, Deng Y, Chandrasekaran K, Mookadam F. Prediction of incipient left ventricular dysfunction in patients with chronic primary mitral regurgitation: a velocity vector imaging study. Eur J Echocardiogr 2011;12:291-298. [PubMed: 21343164]

3. Kazui T, Niinuma H, Tsuboi J, Okabayashi H. Changes in left ventricular twist after mitral valve repair. J Thorac Cardiovasc Surg 2010;141: 716-724. [PubMed: 20541224]

4. Shudo Y, Nakatani S, Sakaguchi T, et al. Left ventricular mechanics following restrictive mitral annuloplasty for functional mitral regurgitation: two-dimensional speckle tracking echocardiographic study. Echocardiography 2012;29:445-450. [PubMed: 22486374]

5. Borg AN, Harrison JL, Argyle RA, Ray SG. Left ventricular torsion in primary chronic mitral regurgitation. Heart 2008;94:597-603. [PubMed: 17881475] 
6. Tibayan FA, Yun KL, Fann JI, et al. Torsion dynamics in the evolution from acute to chronic mitral regurgitation. J Heart Valve Dis 2002;11: 39-46; discussion 46. [PubMed: 11858164]

7. Ennis DB, Nguyen TC, Itoh A, et al. Reduced systolic torsion in chronic "pure" mitral regurgitation. Circ Cardiovasc Imaging 2009;2:85-92. [PubMed: 19808573]

8. Bovendeerd PH, Arts T, Huyghe JM, van Campen DH, Reneman RS. Dependence of local left ventricular wall mechanics on myocardial fiber orientation: a model study. J Biomech 1992;25:1129-1140. [PubMed: 1400513]

9. Young AA, Cowan BR. Evaluation of left ventricular torsion by cardiovascular magnetic resonance. J Cardiovasc Magn Reson 2012;14:49. [PubMed: 22827856]

10. Sengupta PP, Tajik AJ, Chandrasekaran K, Khandheria BK. Twist mechanics of the left ventricle: principles and application. JACC Cardiovasc Imaging 2008;1:366-376. [PubMed: 19356451]

11. Schiros CG, Dell'Italia LJ, Gladden JD, et al. Magnetic resonance imaging with 3-dimensional analysis of left ventricular remodeling in isolated mitral regurgitation: implications beyond dimensions. Circulation 2012;125:2334-2342. [PubMed: 22496130]

12. Guttman MA, Zerhouni EA, McVeigh ER. Analysis of cardiac function from MR images. IEEE Comput Graph Appl 1997;17:30-38. [PubMed: 18509519]

13. Thompson RB, Paterson I, Chow K, et al. Characterization of the relationship between systolic shear strain and early diastolic shear strain rates: insights into torsional recoil. Am J Physiol Heart Circ Physiol 2010;299:H898-H907. [PubMed: 20562339]

14. Reyhan M, Natsuaki Y, Ennis DB. Fourier analysis of stimulated echoes (FAST) for the quantitative analysis of left ventricular twist. J Magn Reson Imaging 2012;35:587-593. [PubMed: 22069227]

15. Khorsand A, Graf S, Frank H, et al. Model-based analysis of electrocardiography-gated cardiac (18)F-FDG PET images to assess left ventricular geometry and contractile function. J Nucl Med 2003; 44:1741-1746. [PubMed: 14602854]

16. Russel IK, Tecelao SR, Kuijer JP, Heethaar RM, Marcus JT. Comparison of 2D and 3D calculation of left ventricular torsion as circumferential-longitudinal shear angle using cardiovascular magnetic resonance tagging. J Cardiovasc Magn Reson 2009;11:8. [PubMed: 19379480]

17. Kotovskaia AR, Koloteva MI, Luk'ianiuk V, Zhernavkov AF, Kondratiuk LL. [Individual prediction by the analysis of preflight ECG data of cardiac function disorders in cosmonauts during standard deorbit after long-term space flights and in the period of postflight observation.] Aerospace Environ Med 2008;42:14-20.

18. Feng W, Nagaraj H, Gupta H, et al. A dual propagation contours technique for semi-automated assessment of systolic and diastolic cardiac function by CMR. J Cardiovasc Magn Reson 2009;11:30. [PubMed: 19674481]

19. Kurjanova EV, Teplyj DL, Zereninova NV. Development of regulation of the cardiac chronotropic function in albino rats during the early postnatal ontogeny according to the results of spectral analysis of heart rhythm variability. Bull Exp Biol Med 2012;152:675-678. [PubMed: 22803162]

20. Tibayan FA, Rodriguez F, Langer F, et al. Alterations in left ventricular torsion and diastolic recoil after myocardial infarction with and without chronic ischemic mitral regurgitation. Circulation 2004;110(11 Suppl 1):II109-114. [PubMed: 15364848]

21. Meral L, Reyhan ML, Gupta H, et al. Left ventricular twist, but not circumferential-longitudinal shear angle, increases with increasing age in normal subjects. J Cardiovasc Magn Reson 2013;15(Suppl 1):P7.

22. Delhaas T, Kotte J, van der Toorn A, Snoep G, Prinzen FW, Arts T. Increase in left ventricular torsion-to-shortening ratio in children with valvular aortic stenosis. Magn Reson Med 2004;51:135-139. [PubMed: 14705053]

23. Fischer SE, McKinnon GC, Maier SE, Boesiger P. Improved myocardial tagging contrast. Magn Reson Med 1993;30:191-200. [PubMed: 8366800] 
Normal

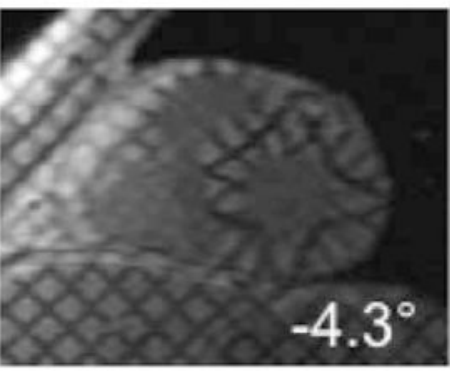

Base

Apex

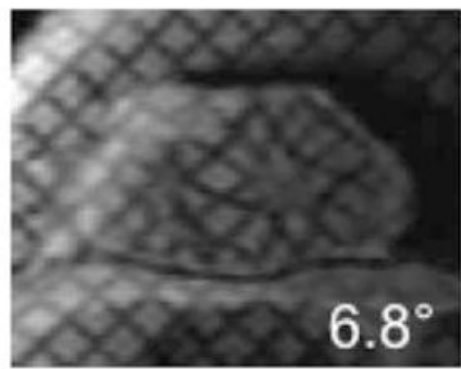

$11.1^{\circ}$

\section{Peak Twist}

\section{Moderate MR}
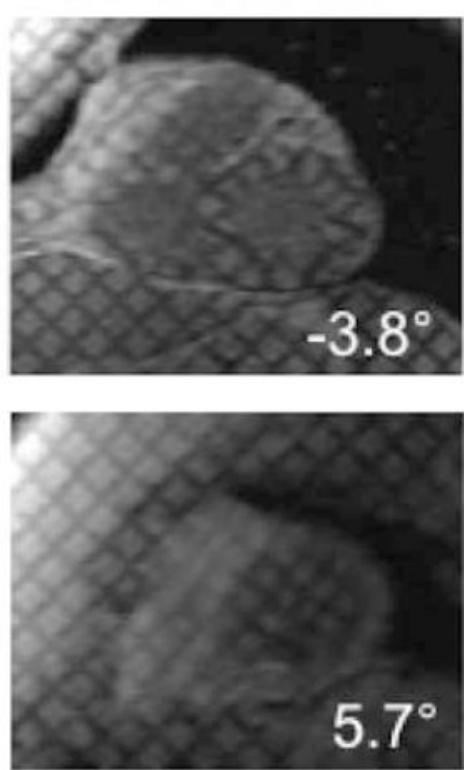

$9.5^{\circ}$
Severe MR
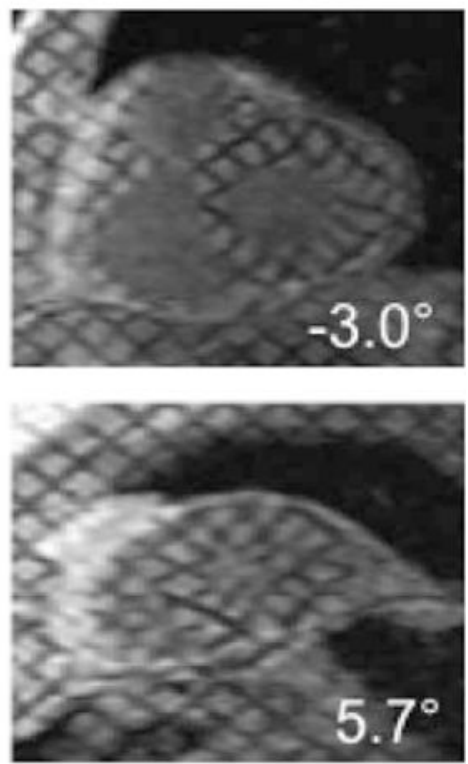

$8.7^{\circ}$

FIGURE 1:

Representative tagged images from a normal subject and patients with moderate MR and severe MR. The basal and apical frames capture the time of peak LV twist. Individual LV rotation values are noted within the frame and peak LV twist values below the frame. 


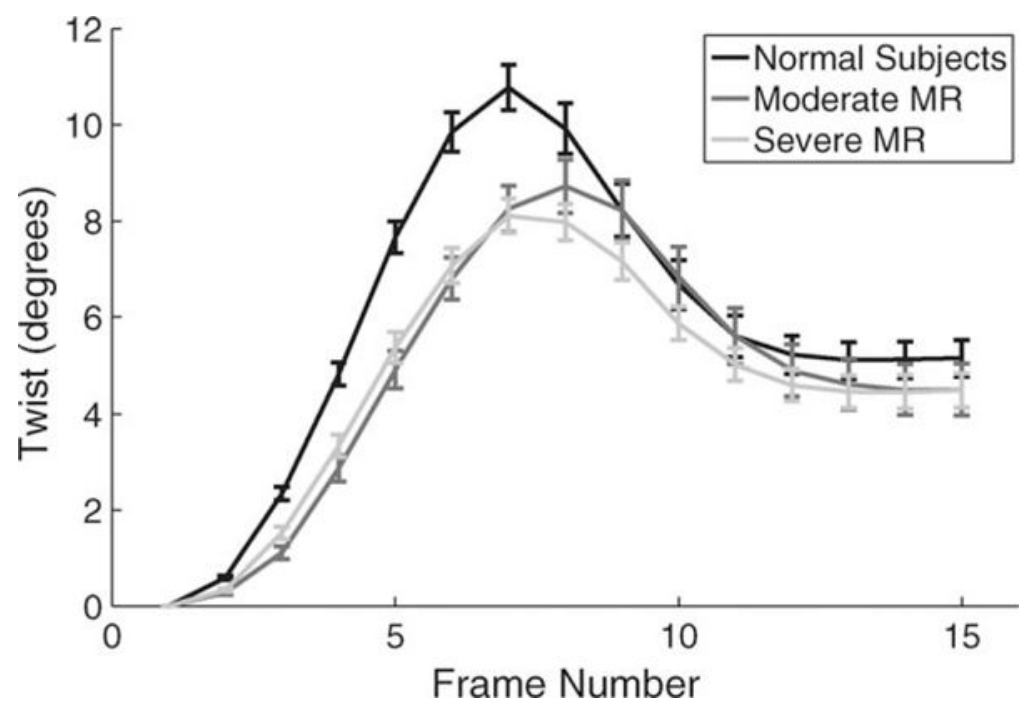

FIGURE 2:

Population mean LV twist during the cardiac cycle for normal subjects and patients with moderate MR and severe MR. Error bars are standard error of the mean for clarity. Mean peak LV twist in normal subjects is larger compared to both patients with moderate and severe MR $(P=0.0001)$. No significant difference in mean peak LV twist was observed between moderate and severe MR patients (Table 2). 


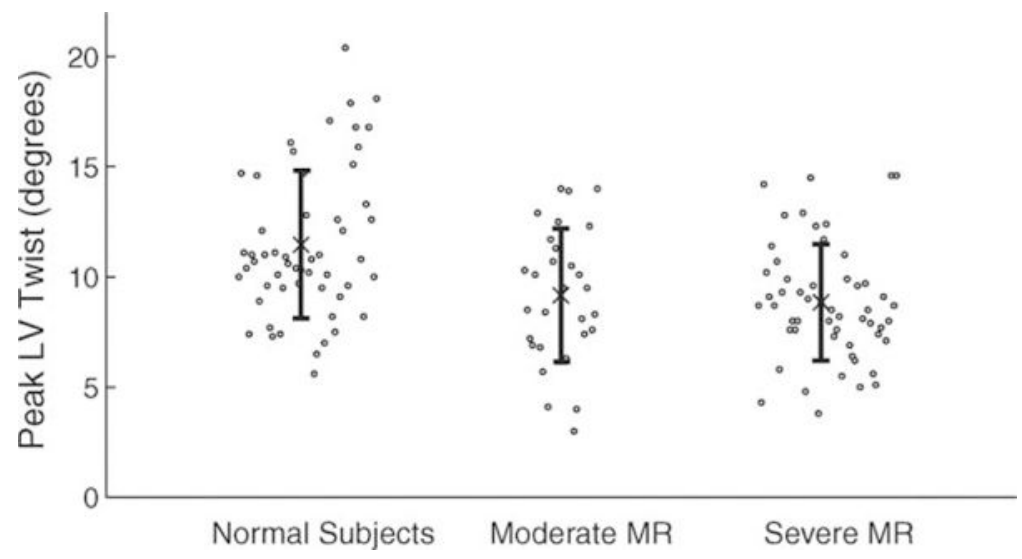

FIGURE 3:

Peak LV twist values for normal subjects, moderate MR subjects, and severe MR subjects. The " $X$ " represents the mean peak LV twist and the error bars represent the standard deviation. LSD only showed significant decreases between the normal subjects and the moderate MR patients and between the normal subjects and the severe MR patients and no difference between the moderate and severe MR patients. 


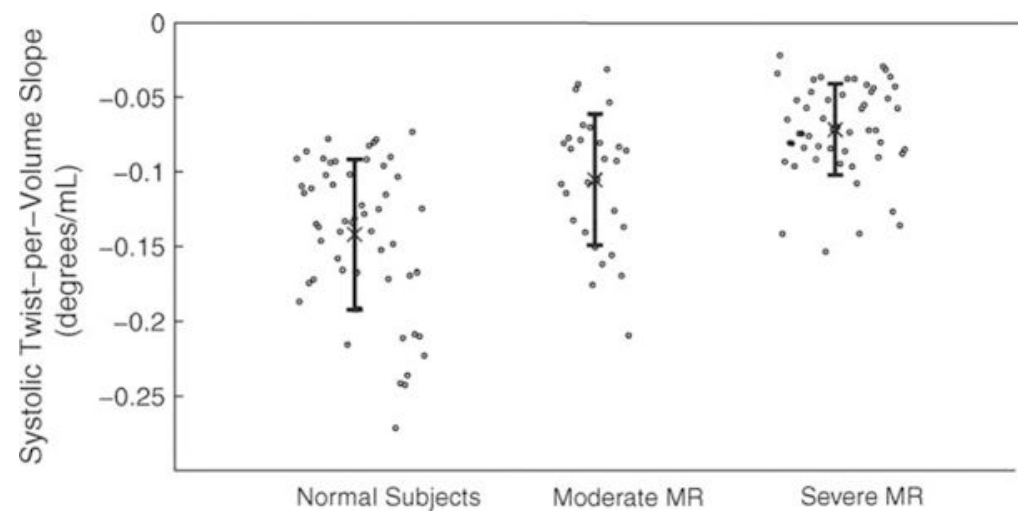

FIGURE 4:

Systolic twist-per-volume slope derived from normal subjects, moderate MR subjects, and severe MR subjects. The "X" represents the mean systolic twist-per-volume slope and the error bars represent the standard deviation. LSD detected significant decreases in systolic twist-per-volume slope between normal subjects and moderate MR patients, normal subjects and severe MR patients, and moderate MR patients and severe MR patients. 


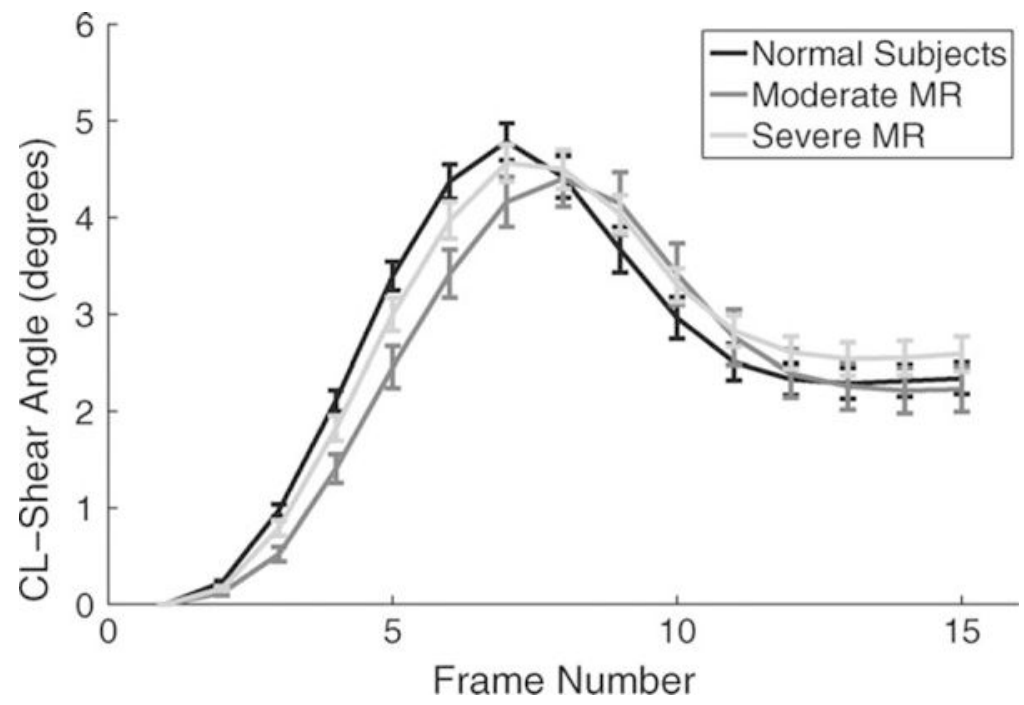

FIGURE 5:

CL-shear angle for normal subjects and patients with moderate MR and severe MR during the cardiac cycle. Data curves represent the population mean. Standard error of the mean is used for the error bars for clarity. The magnitude of LV CL-shear angle appears to pseudonormalize $(P=0.7)$. 


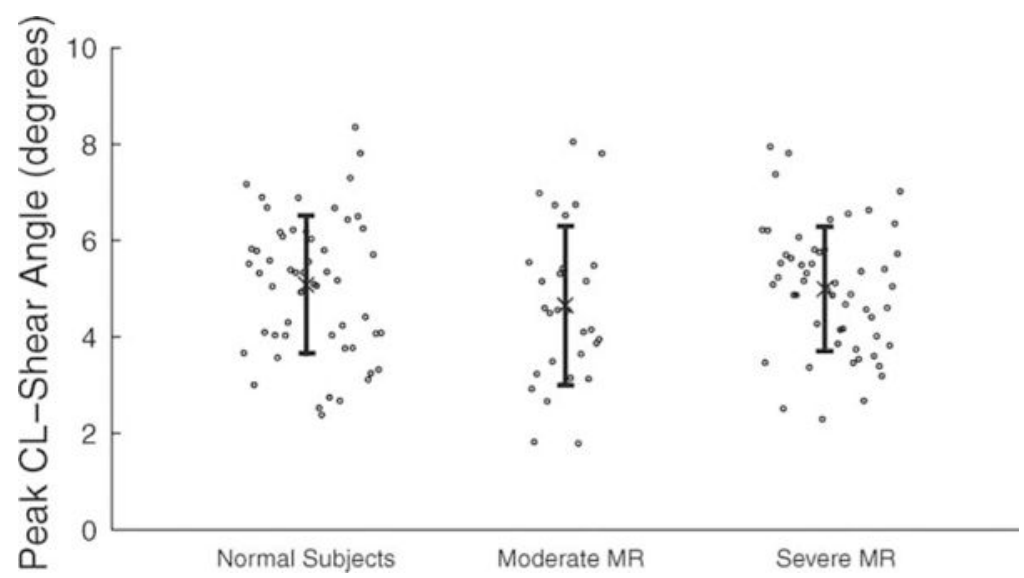

FIGURE 6:

Peak LV CL-shear angle for normal subjects, moderate MR subjects, and severe MR subjects. The "X" represents the mean peak CL-shear angle and the error bars represent the associated standard deviation of the mean. All pairwise comparisons of peak CL-shear angle for normal, moderate MR, and severe MR were considered not significant 


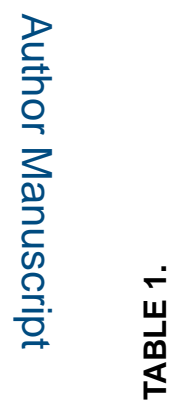

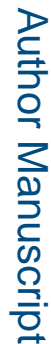

峁

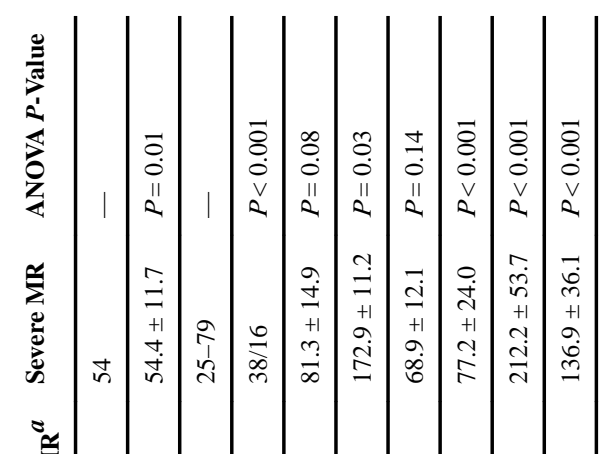

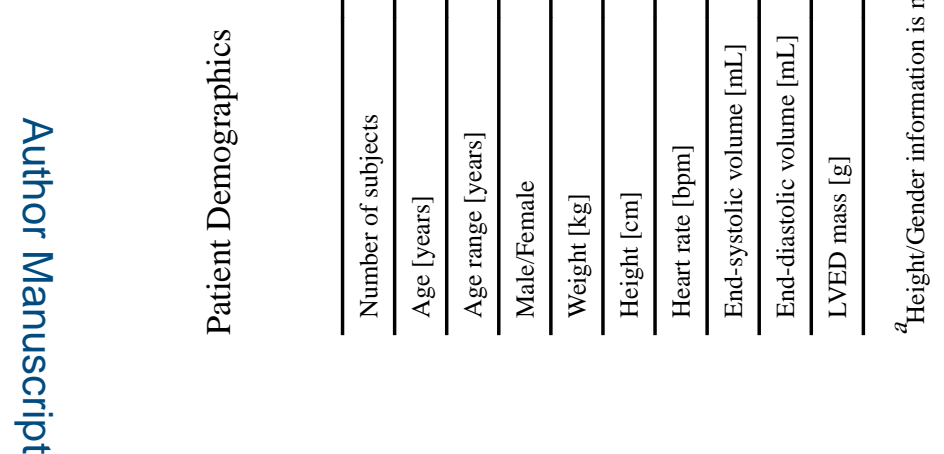
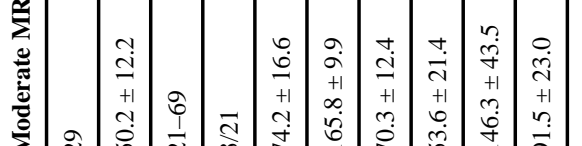

$\frac{\dot{s}}{\stackrel{0}{0}}$

य) क ल

$n$
$\infty$
0
0

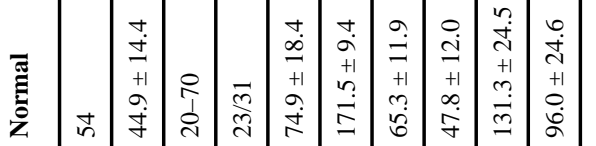

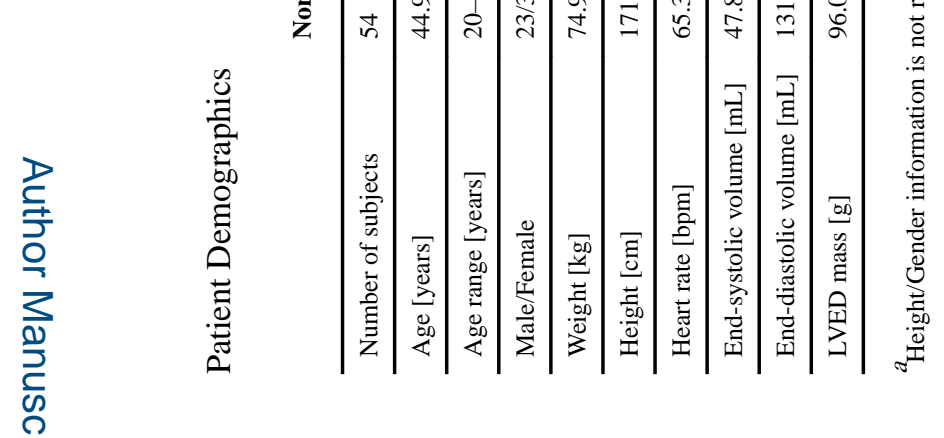
JMagn Reson Imaging. Author manuscript; available in PMC 2019 March 25. 


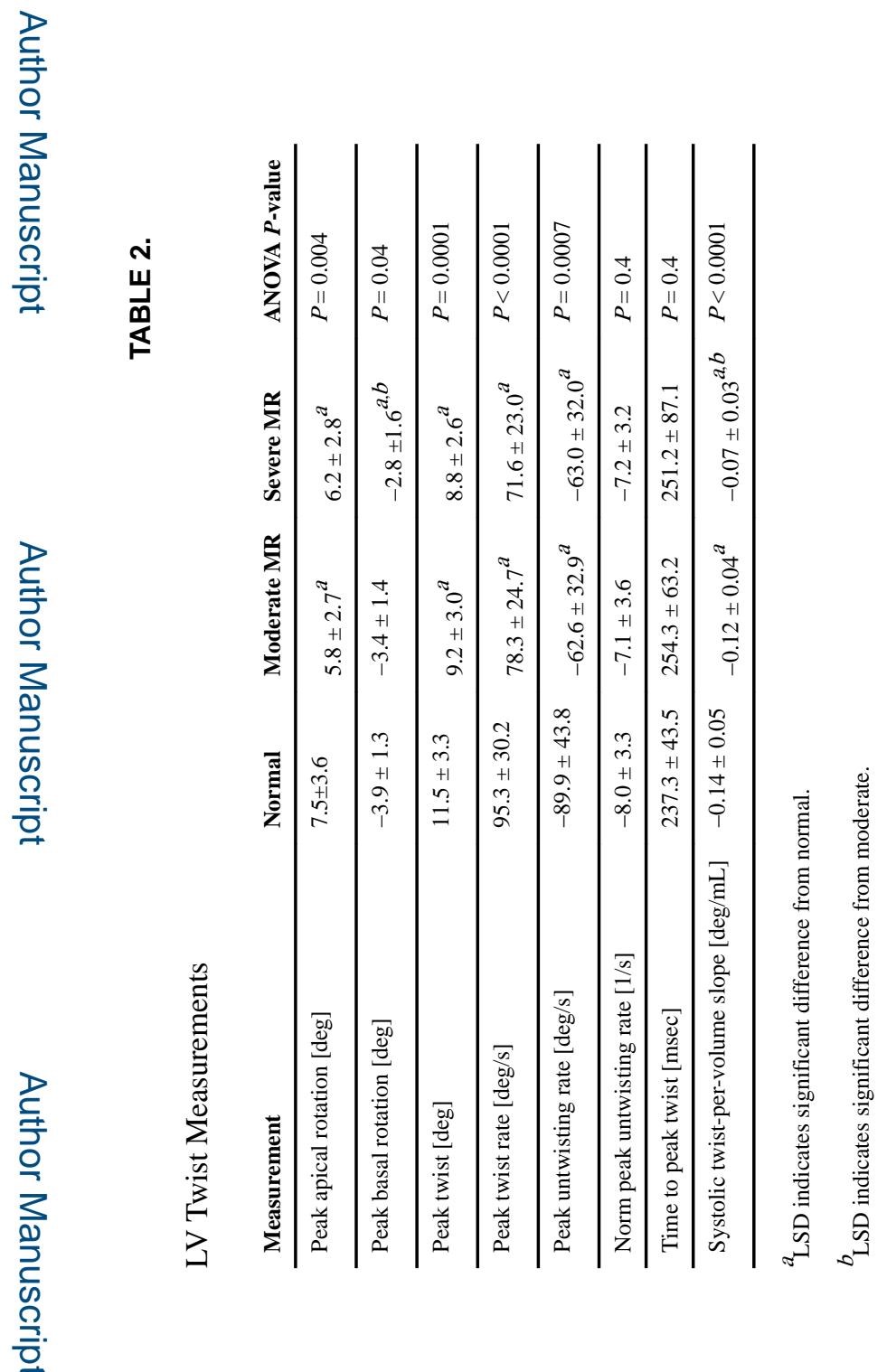




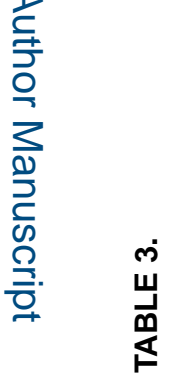

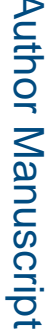

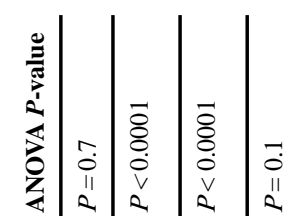

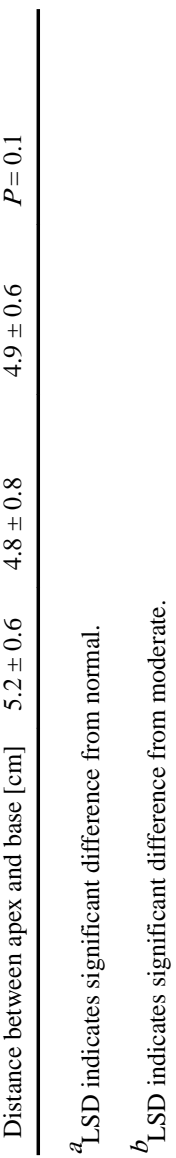

